\title{
Innovation and Opportunities in Pediatric Therapeutic Development
}

\author{
Therapeutic Innovation \\ \& Regulatory Science \\ 2019, Vol. 53(5) 564-566 \\ (C) DIA 2019 \\ Article reuse guidelines: \\ sagepub.com/journals-permissions \\ DOI: 10.1 I77/2168479019869754 \\ tirs.sagepub.com
}

In 2015, Spielberg and Portman introduced the issue in TIRS on "New Horizons in Pediatric Drug Development." 1 The issue articulated the challenges and opportunities in pediatric research and therapeutic development for children and highlighted the need for collaborative approaches and the inclusion of the patient perspective. This issue of TIRS updates the state of pediatric therapeutic development with an eye to the future. Much progress has been made, but we continue to face challenges and opportunities that need to be addressed through basic and clinical research, innovation, and collaboration.

The field of pediatrics has been recognized since ancient times, including references from the works of Hippocrates. Use of medications in children has a long and storied past from exposure to tetanus tainted diphtheria toxin, through the sulfanilamide pediatric formulation tragedy and the in utero exposure to thalidomide. Each of these tragedies resulted in legislation to encourage the development of safe and efficacious therapeutics. Following the 1962 Kefauver-Harris Amendments to the 1938 Food, Drug, and Cosmetic Act, inclusion of children in human drug trials was considered too risky, leaving them as "therapeutic orphans," a term coined by Harry Shirkey. It wasn't until 1997 that the first legislation to encourage pediatric drug development was passed under the FDA Modernization Act (FDAMA). Subsequent legislation provided an incentive and a requirement for pediatric drug ${ }^{i}$ development through the Best Pharmaceuticals for Children Act (BPCA) of 2002 and the Pediatric Research Equity Act (PREA) of 2003, respectively. Both programs were made permanent in 2012 under the Food and Drug Administration Safety and Innovation Act (FDASIA). These legislative efforts at encouraging pediatric studies have been successful, and as of April 2019, there have been 785 new pediatric labeling changes because of the combination of BPCA and PREA. However, there are challenges in developing pediatric therapeutics, especially in rare diseases, including gaps in understanding the basic science of disease as well as limitations in the knowledge of the mechanism of action of therapeutic products and the ontogeny of disease manifestations and drug absorption, distribution, metabolism, and excretion. Many diseases, especially in the rare disease space, occur primarily in children, thus posing additional ethical, methodological, and operational challenges. Whenever possible, it is critically important to leverage information from multiple additional sources including data from adults or other pediatric patient subgroups as well as modeling and simulation based on available pharmacokinetic (PK) and pharmacodynamic (PD) data. Creative approaches to obtaining pediatric data can include innovative trial designs, Bayesian statistical analyses, and use of real-world data to supplement clinical trial information. More efficient use of resources can be accomplished through the development of global pediatric clinical trial networks.

In this special pediatric issue of $T I R S$, there are a series of articles focused on scientific approaches to obtaining data in pediatric therapeutic development, processes to facilitate the generation of pediatric data, inclusion of all the stakeholders to optimize pediatric therapeutic development, and policy and legislative approaches to encourage the development of pediatric therapeutics. With the initiation of BPCA and PREA, there was an impetus to develop pediatric therapeutics. Given the experience over the past 15 years, it is now time to refine the approach to optimize the development of pediatric data to support safety and efficacy labeling of pediatric therapeutics.

Several articles in this issue address the creative approaches to data generation to support pediatric labeling. Gamalo et al note that there is an ethical obligation to only enroll pediatric patients in a clinical trial if there is no other way to obtain the data, and an imperative to leverage data from studies in adults and other pediatric subgroups (extrapolation), as well as developing creative analytic strategies including modeling and simulation, and Bayesian statistical analyses. ${ }^{2}$ When the course of a disease and a drug's effects are sufficiently similar in the pediatric and adult populations, extrapolation should be strongly considered, and if possible, may need to be supplemented by pediatric PK, PD, and safety data. Additional sources of data may include innovative trial designs and real world data to support clinical trial information. Additionally, important information can be obtained from studies in conditions where a similar mechanism of disease and response to medication are seen, for example, in many immunologic diseases. It is important that pediatric development be considered during the planning of adult trials so that biomarker and exposure response data can be obtained in adults and then used to bridge to the pediatric studies. Liu et al introduce the concept of the use of pharmacometrics to guide the selection of drug doses for pediatric patients, including a discussion of nonlinear mixed effects modeling (NONMEM) that is useful with sparse data, as this may be particularly applicable in pediatric studies. ${ }^{3}$ Additional mechanistic models including physiologically based pharmacokinetics and clinical trial simulations are discussed. It is important that clinicians understand how to analyze the data obtained through these modeling approaches. Children are a heterogeneous population, and the ontogeny of enzyme and organ systems, as manifested by age-related rapid changes in PK and PD, plays a critical role in disease pathophysiology and 
response to therapy. Job et al discuss the maturation of systems associated with absorption, distribution, metabolism, and elimination. ${ }^{4}$ Newborns, especially preterm infants, are the most vulnerable to these ontogenic changes and it is important to take into consideration not only gestational age at birth but postnatal age as many of the enzyme systems mature differentially based on both gestational age and postnatal age. Conversely, it is becoming more apparent that adolescents have very similar responses to drugs at equivalent doses to adults, making the inclusion of adolescents in adult trials in the absence of concerning safety signals both feasible and efficient. How early in the drug development process this should occur depends on the pathophysiology of the disease and the benefit-risk assessment of the drug mechanism of action.

All the innovative approaches outlined above have focused primarily on efficacy assessments. Pediatric safety monitoring is critically important. While pediatric safety data cannot be solely determined by extrapolation, safety data from adults and other pediatric subgroups can be leveraged in the analysis but specific safety parameters should be studied prospectively to fully understand the breadth and depth of any potential safety issues. In general, growth and development are key aspects of pediatric safety monitoring that may require longer-term follow-up. Hirschfeld and Zajicek discuss the process for data capture and analysis of potential safety signals with a focus on assessments, terminology, data formats, and structure that support the next generation of data systems, processes, and analyses. $^{5}$

In order to be successful in the generation of pediatric data, it is critical that processes be established to facilitate pediatric clinical trial implementation. In an upcoming TIRS issue, Turner et al will describe the development of global pediatric network capabilities. ${ }^{6}$ Currently, clinical research networks are under development in the US, Europe, Japan, and Canada in a global interoperative effort to streamline the conduct of multiple pediatric clinical trials. It is important that these networks develop sustainability. As part of the clinical trial network approaches, it is critical that all the stakeholders are included in these efforts. Representatives from academia, industry, regulatory agencies, and patient/parent advocacy groups are key stakeholders in developing clinical protocols. FDA has taken an active role in including the patient voice in drug development and evaluation. Tsang et al discuss the importance of understanding that the pediatric voice needs to be heard. ${ }^{7}$ Pediatric patients are powerful advocates who are altruistic and eager to support other patients who are dealing with medical conditions. They are particularly knowledgeable about endpoints that are clinically meaningful, and several pediatric advocacy groups are including both parents and pediatric patients in the design of clinical trials. Clearly, there is an ongoing need to increase the collaborative efforts between all stakeholders with a focus on incorporating the voice of pediatric patients and their families.

The legislative incentives and mandates were important to spur development of pediatric therapeutics, but it is important to understand where they have been successful and where there might be room for improvement. Yen et al performed a survey to assess the impact of the regulatory incentive programs. ${ }^{8}$ They identified a number of ongoing challenges including the availability of clinically meaningful pediatric endpoints, the ability to identify pediatric patients to enroll in clinical trials, the willingness and capability of investigators to share data, and the capability of sponsors to develop pediatric specific formulations. The neonatal population continues to be one that has been particularly difficult to study. In addition to the ontogenic issues complicating neonatal PK/PD studies, most of the diseases in neonates are unique to that population so that BPCA and PREA may not be particularly relevant. It has been difficult to spur therapeutic development in neonates. Bucci-Rechtweg et al propose policy reform to incentivize drug development and fund research in neonatal-specific diseases. ${ }^{9}$

Great progress in labeling drugs for pediatric patients has been made in the past 15 years following the passage of BPCA and PREA. However, there continues to be substantial off-label use of drugs in children, especially neonates, and pediatric drug development lags behind development in adults. In addition, several pediatric drug trials have failed even in the face of positive trials in adults for the same drug. It is not clear whether this is a true therapeutic failure or secondary to issues with identifying appropriate dosing and/or endpoints. In addition, there are ongoing needs for safety assessments for all pediatric therapeutics that are being developed. Pediatric drug development has been inefficient-improved efficiency will require active involvement of all stakeholders in a collaborative effort to understand disease pathophysiology and drug mechanism of action, and to complete pediatric clinical trials. Patients/parents will need to be involved in protocol development as they can provide key information with respect to clinically meaningful endpoints, and the stakeholder community will need to work to confirm the sensitivity and specificity of short-term and longterm endpoints. Collaboration through global clinical trial networks can facilitate access to patients and resource utilization. Now that the medical community has established that it is possible to develop pediatric therapeutics, there is a public health requirement to ensure that efficacy and safety are determined for all drugs that are prescribed for children.

Susan McCune, MD

Director, Office of Pediatric Therapeutics Office of Clinical Policy and Programs Office of the Commissioner U.S. Food and Drug Administration

Ronald J. Portman, MD, FAAP, FASN, FASH Executive Director, Pediatric Development, Science and Innovation Pediatric Center of Excellence Clinical Development \& Analytics, Novartis Pharmaceuticals Corporation 


\section{Author Note}

The opinions expressed in this article are those of the authors and should not be interpreted as the position of the US Food and Drug Administration.

\section{Note}

i. Drug in this article refers to drugs and biologics.

\section{References}

1. Spielberg SP, Portman R. New horizons in pediatric drug development. Therapeutic Innovation \& Regulatory Science. 2015;49(5):613-614.

2. Gamalo-Siebers M, Hampson L, Kordy K, et al. Incorporating innovative techniques toward extrapolation and efficient pediatric drug development. Therapeutic Innovation \& Regulatory Science. 2019. doi:10.1177/2168479019842541

3. Liu X, Ward RM. Pharmacometrics in pediatrics. Therapeutic Innovation \& Regulatory Science. 2019. doi:10.1177/216847901 9851793
4. Job K, Gamalo-Siebers M, Ward RM. Pediatric age groups and approach to studies. Therapeutic Innovation \& Regulatory Science. 2019. doi: $10.1177 / 2168479019856572$

5. Hirschfeld S, Zajicek A. What could the future of safety monitoring look like? Therapeutic Innovation \& Regulatory Science. 2019. doi: 10.1177/2168479019854339.

6. Turner M, Benjamin D, Hovinga C, et al. Pediatric Clinical Research Networks to accelerate development of therapeutics in all pediatric specialties and age groups. Therapeutic Innovation \& Regulatory Science. (in prep).

7. Tsang VWL, West L, Woods C, et al. Role of patients and parents in pediatric drug development. Therapeutic Innovation \& Regulatory Science. 2019. doi: 10.1177/2168479018820875.

8. Yen E, Davis JM, Milne CP. Impact of regulatory incentive programs on the future of pediatric drug development. Therapeutic Innovation \& Regulatory Science. 2019. doi:10.1177/2168479019837522

9. Bucci-Rechtweg CM, Ward RM. Tiny and forgotten: a call for focused neonatal policy reform. Therapeutic Innovation \& Regulatory Science. 2019. doi:10.1177/2168479018821922 